\title{
ADICIONES AL CONOCIMIENTO DE LA DIVERSIDAD DE LOS HONGOS ANAMORFOS DEL BOSQUE MESÓFILO DE MONTAÑA DEL ESTADO DE VERACRUZ III
}

\author{
Rosa María Arias ${ }^{1}$, Gabriela Heredia ${ }^{1}$ y Julio Mena-Portales ${ }^{2}$ \\ ${ }^{1}$ Instituto de Ecología, A.C., km 2.5 antigua carretera a Coatepec 351 \\ Colonia Congregación el Haya. 91070 Xalapa, Veracruz, México. \\ rosa.arias@inecol.edu.mx \\ ${ }^{2}$ Instituto de Ecología y Sistemática, carretera de Varona km 3.5 \\ Apdo. postal 8029, Capdevila, Boyeros. La Habana, Cuba.
}

\section{RESUMEN}

Esta contribución es parte de una serie de artículos cuyo objetivo es incrementar el conocimiento de los hongos anamorfos saprobios que proliferan en el bosque mesófilo de montaña en el estado de Veracruz. Se presentan las descripciones e imágenes de 25 especies. Todas ellas, excepto Helminthosporium solani, son nuevos reportes para México. Dictyochaeta tumidospora, Fusariella intermedia, Hyphodiscosia queenslandica y Dictyosporium digitatum no habían sido registradas desde su descripción original. Se incluyen datos de su distribución y sustratos en los que se han colectado.

Palabras clave: bosque mesófilo de montaña, hongos anamorfos, hongos conidiales, hongos saprobios, Hyphomycetes, México, Veracruz.

\begin{abstract}
As part of a series of papers dealing with the diversity of anamorphic saprophytic fungi in cloud forest remnants from the Veracruz state, an account of 25 species are described and illustrated. All of them but Helminthosporium solani are new records for the Mexican mycobiota. The species Dictyochaeta tumidospora, Fusariella intermedia, Hyphodiscosia queenslandica and Dictyosporium digitatum had not been recorded since their original description. For all taxa, data about their geographical distribution and substrates are included.
\end{abstract}


Key words: anamorphic fungi, cloud forest, conidial fungi, Hyphomycetes, Mexico, saprobic fungi, Veracruz.

\section{INTRODUCCIÓN}

El bosque mesófilo de montaña (BMM) es uno de los ecosistemas más interesantes e importantes de México por su alta diversidad vegetal y elevado número de endemismos, características que se derivan de las particulares condiciones climatológicas en donde prospera (Williams-Linera, 2007). Aun cuando en los últimos años han aumentado las aportaciones sobre la riqueza de especies de los hongos anamorfos en este tipo de vegetación (Delgado-Rodríguez et al., 2006; Heredia y Reyes, 1999, Heredia et al., 2004 y Heredia et al., 2006), queda mucho por hacer al tratarse de un grupo con una amplia diversidad. Ante la acelerada transformación de las escasas áreas que prevalecen con BMM en el país, resulta apremiante la realización de constantes exploraciones de campo para la detección e identificación de las especies anamorfas que se desarrollan en las espesas capas de hojarasca acumuladas a nivel del suelo en este tipo de vegetación.

Como resultado de más de una década de trabajo de campo y de orden taxonómico, surge la presente serie de artículos que persigue además de dar a conocer las especies anamorfas saprobias que crecen sobre los restos vegetales del BMM, incrementar el conocimiento para México de este grupo de hongos que, a pesar de su abundancia y alta diversidad, había sido ignorado por los micólogos.

\section{MÉTODOS}

El material examinado consistió en restos vegetales colectados en áreas con manchones de vestigios de BMM localizadas en el centro del estado de Veracruz (Williams-Linera, 2007); la mayor parte proviene del Rancho Guadalupe y del Jardín Botánico Javier Clavijero, ambos localizados en las afueras de la ciudad de Xalapa en el km 2.5 de la carretera antigua Xalapa-Coatepec. En el laboratorio se colocó el material biológico en cámaras húmedas. Diariamente, con un microscopio estereoscópico se efectuaron revisiones para extraer las estructuras de reproducción y elaborar preparaciones permanentes y semipermanentes, que se encuentran depositadas en la colección de hongos anamorfos del Instituto de Ecología, A.C. en Xalapa, Veracruz (XAL). La identificación de las especies y las descripciones que 
se presentan se realizaron con base en las estructuras fúngicas que se desarrollan in substratum.

\section{RESULTADOS}

Se definen las descripciones de 25 especies identificadas, 24 de las cuales representan el primer registro para México. Los hongos Cheiromyceopsis echinulata, Dictyochaeta tumidospora, Fusariella intermedia e Hyphodiscosia queenslandica se consideran elementos poco comunes debido a que a nivel mundial han sido registrados en muy pocas áreas geográficas.

Las especies Dictyochaeta tumidospora, Fusariella intermedia, Hyphodiscosia queenslandica y Dictyosporium digitatum constituyen primeros registros para el Neotrópico. Para todas se incluyen datos de su distribución y sustratos en los que han sido colectadas.

Bactrodesmium spilomeum (Berk. \& Broome) E.W. Mason \& S. Hughes, Can. J. Bot. 31(5): 616 (1953). (Fig. 1).

三Sporidesmium spilomeum Berk. \& Broome. apud Rabenhorst, Fungi Europaei No. 1162 (1868).

Colonias compuestas por esporodoquios esparcidos sobre el sustrato. Esporodoquios punctiformes, cafés oscuros, de 100-300 $\mu \mathrm{m}$ de diámetro. Conidióforos micronematosos, en fascículos, erectos, flexuosos, frecuentemente ramificados, pared lisa, septados, subhialinos, hasta de $40 \mu \mathrm{m}$ de largo, de 2-3 $\mu \mathrm{m}$ ancho en la base y alrededor de $4.5 \mu \mathrm{m}$ en el ápice. Células conidiógenas monoblásticas, integradas, terminales, determinadas, cilíndricas. Conidios solitarios, acrógenos, elipsoidales a claviformes, redondeados en el ápice, base truncada, pared lisa, 2-5(-4) septos, cafés a cafés pálidos, de 39.2-48.5 × 14.7-19.6 $\mu \mathrm{m}$, aproximadamente con $5.8 \mu \mathrm{m}$ de ancho en la base.

Material estudiado: ramas muertas, col. J. Mena-Portales, 13/06/95, CB845. Rancho Guadalupe, mpio. Xalapa.

Distribución y sustratos: se ha colectado sobre Betula, Fagus sylvatica, Fraxinus excelsior, Quercus pubescens y corteza de Ulmus campestris en Inglaterra (Ellis, 1959), sobre Populus tremuloides en Canadá (Sutton, 1973), también se le ha encontrado creciendo sobre Bambusa sp. en China (Lu et al., 2000; Zhuang, 2001). 
Brachysporium obovatum Keissl., Syll. fung. (Abellini) 4: 427 (1886). (Fig. 3).

Colonias dispersas, cafés oscuras a negras. Conidióforos macronematosos, solitarios o en grupos, erectos o suberectos, rectos a ligeramente flexuosos, simples, subulados, pared lisa, septados, cafés oscuros, ápice más pálido, hasta de $390 \mu \mathrm{m}$ de largo, aproximadamente con $20 \mu \mathrm{m}$ de ancho en la base hinchada y $10 \mu \mathrm{m}$ justo por encima de la célula basal. Células conidiógenas poliblásticas, integradas, terminales, determinadas, simpodiales, cilíndricas, denticuladas. Conidios solitarios, acropleurógenos, ovoides o piriformes, pared lisa, 2 septos, de 17.5-21 $\times$ 9-9.5 $\mu \mathrm{m}$, cada conidio presenta en la base una célula separadora cilíndrica, hialina a subhialina, recta o curva.

Material estudiado: tronco muerto, col. J. Mena-Portales, 13/06/95, CB398 y CB410-3. Rancho Guadalupe, mpio. Xalapa.

Distribución y sustratos: esta especie es común encontrarla sobre madera podrida y en corteza de diversos árboles. Se ha colectado sobre Fagus sp., Populus sp., Prunus avium, Platanus sp. y Quercus sp. en Alemania e Inglaterra (Ellis, 1971; Dennis, 1986); en Alnus glutinosa, Betula verrugosa, Carpinus betulus, Fagus sylvatica, Fraxinus excelsior, Picea sp. Prunus avium, Quercus petrea, Q. robur y Tilia cordata en la antigua Checoslovakia (Holuvobá-Jechová, 1972); creciendo en Fagus crenata y Quercus sp. en Japón (Matshushima, 1975); en madera podrida de Fagus sylvatica en Hungría (Holuvobá-Jechová, 1979, Revay, 1986); también se ha colectado en Betula alleghaniensis en Canadá (Ginns, 1986); sobre hojas de Eucalyptus sp. en California (Tidwell, 1990); sobre Quercus pubescens en Rusia (Melnik y Popushoi, 1992) y sobre un tronco muerto en España (Mena-Portales et al., 2000).

Chalara aspera (Piroz. \& Hodges) P.M. Kirk, Kew Bull. 38(4): 580 (1984). (Fig. 4). 三Chaetochalara aspera Piroz. \& Hodges, Can. J. Bot. 51(1): 157 (1973).

Teleomorfo: Calycellina carolinensis Nag Raj \& W.B. Kendr., A monograph of Chalara and allied genera. Pág. 183 (1975).

Colonias dispersas, aterciopeladas, café grisáceas. Setas solitarias, rectas, subuladas, adelgazándose hacia el ápice, pared gruesa, septadas, cafés oscuras, hasta de $325 \mu \mathrm{m}$ de largo, aproximadamente de $7.5 \mu \mathrm{m}$ de ancho en la base. Conidióforos macronematosos, solitarios o agrupados alrededor de la seta, erectos, rectos, simples, cilíndricos o ampuliformes, pared verrugosa cerca del ápice, 1 septo, café a café dorado pálidos, hasta de $100 \mu \mathrm{m}$ de largo, alrededor de $7 \mu \mathrm{m}$ de ancho en la parte más amplia y $4 \mu \mathrm{m}$ cerca del ápice. Células conidiógenas monofialídicas, inte- 
gradas, terminales. Conidios catenulados, endógenos, cilíndricos, truncados en las extremos, pared lisa, 1 septo, hialinos, de 17-19 $\times 2 \mu \mathrm{m}$.

Material estudiado: hojas de Macadamia sp., col. G. Heredia, 10/06/95, CB846. Rancho Guadalupe, mpio. Xalapa.

Distribución y sustratos: existen registros sobre hojas caídas de Persea borbonia en Estados Unidos (Pirozynski y Hodges, 1973) y sobre Knightia excelsa y Myrica cerifera en Nueva Zelanda (Nag Raj y Kendrick, 1975).

Cheiromyceopsis echinulata Mercado \& J. Mena, Acta Bot. Cubana 53: 2 (1988). (Fig. 2).

Colonias formadas por esporodoquios. Esporodoquios convolutos, cafés oscuros, de 80-300 $\mu \mathrm{m}$ de diámetro. Conidióforos macronematosos, erectos, rectos o flexuosos, mayormente sin ramificar, pared lisa, septados, cafés pálidos o café oliváceos. Células conidiógenas monoblásticas, integradas, terminales, determinadas, cilíndricas. Conidios solitarios, acrógenos, en forma de guante, compuestos por una célula basal pedicelada de la que surgen 3-10 (usualmente 5-8) hileras de células, pared equinulada, 2-7 septos, cafés oscuros, de 14.5-21.5 × 10-12.5 $\mu \mathrm{m}$; las hileras independientes miden $6 \mu \mathrm{m}$ de grosor.

Material estudiado: corteza de Liquidambar sp., col. J. Mena-Portales, 13/06/95, CB848. Rancho Guadalupe, mpio. Xalapa.

Distribución y sustratos: Cheiromyceopsis echinulata sólo había sido registrada creciendo sobre pecíolo y raquis de Roystonea regia en Cuba (Mercado-Sierra y Mena-Portales, 1986; Delgado-Rodríguez et al., 2002; Delgado-Rodríguez y MenaPortales, 2004).

Chloridium virescens var. chlamydosporum (J.F.H. Beyma) W. Gams \& Hol.Jech., Stud. Mycol. 13: 21 (1976). (Fig. 5).

三Bisporomyces chlamydosporus J.F.H. Beyma, Antonie van Leeuwenhoek 6: 277 (1940).

三Chloridium chlamydosporum (J.F.H. Beyma) S. Hughes, Can. J. Bot. 36: 748 (1958).

Colonias dispersas, velutinas, café grisáceas a cafés oscuras. Conidióforos macronematosos, solitarios, erectos, rectos, simples, pared lisa, 3-7 septos, cafés a cafés oscuros en la base, hasta de $130 \mu \mathrm{m}$ de largo, de 3-4 $\mu \mathrm{m}$ de ancho. Células conidiógenas monofiálidicas, integradas, terminales, percurrentes, simpodiales, cilíndricas, 
con un collarín en forma de embudo, cafés pálidas. Conidios agregados en cabezuelas mucilaginosas, semiendógenos, elipsoidales, hialinos, de 3-5 × 1.5-2.5 $\mu \mathrm{m}$.

Material estudiado: tronco muerto de Pinus sp. col. J. Mena-Portales, 21/07/95, CB477-1. Xicochimalco, mpio. Xicochimalco; sobre ramas muertas, col. J. MenaPortales, 28/07/95, CB847, 847-1. Rancho Guadalupe, mpio. Xalapa.

Distribución y sustratos: este hongo ha sido colectado en diferentes sustratos como troncos caídos, suelo volcánico, suelo de bosque, suelo de cultivo de maíz, bajo madera de arce, sobre hojas de Betula verrucosa, Cedrus atlantica, Fraxinus excelsior, Populus nigra, Populus sp., Picea sp., Quercus sp., Quercus robur, Sorbus aucuparia y Urtica dioica en África, Bélgica, Canadá, la antigua Checoslovakia, Chile, Estados Unidos, Francia, Holanda, Suecia (Gams y Holubová-Jechová, 1976); en ramas caídas de Carpinus betulus y Fagus sylvatica en Hungría (Holubová-Jechová, 1979); creciendo en acículas de Pinus sp. en Tailandia (Tokumasu et al., 1990); en raíces de Calathea zebrina, hojas de Euterpe edulis y Maranta bicolor en Brasil (Grandi, 1991, 1999; Grandi y Gusmao, 1996); sobre hojas de Betula pendula en Rusia (Melnik y Popushoi, 1992); también ha sido aislado en ramas muertas de árboles no identificados en Cuba (Mercado-Sierra et al., 1997) y en hojas de Carpinus betulus en Polonia (Chlebicki y Chmiel, 2006).

Dendryphiopsis arbuscula (Berk. \& M.A. Curtis) S. Hughes, Can. J. Bot. 36: 762 (1958). (Fig. 6).

$\equiv$ Helminthosporium arbuscula Berk. \& M.A. Curtis, Grevillea 3: 103 (1875).

Colonias dispersas, cafés oscuras a negras. Conidióforos macronematosos, cespitosos, erectos, rectos, usualmente con algunas ramas cerca de ápice, hasta de $414 \mu \mathrm{m}$ de largo, de 8-13 $\mu \mathrm{m}$ de ancho. Células conidiógenas monotréticas, integradas, terminales, determinadas, cilíndricas. Conidios solitarios, acrógenos, fusiformes a obclaviformes, pared lisa, 3-7 septos, negro oliváceos, de 49-75 × 10-15 $\mu \mathrm{m}$.

Material estudiado: ramas muertas, col. G. Rosas, 13/06/95, CB417-2. Rancho Guadalupe, mpio. Xalapa.

Distribución y sustratos: se ha reportado sobre ramas muertas de Acer, Carya, Magnolia y Rhus en Estados Unidos (Ellis, 1976); también existen registros de su presencia sobre madera de Dacrydium cupressinum y Quintinia serrata en Nueva Zelanda (Hughes, 1978b).

Dictyochaeta tumidospora Kuthub. \& Nawawi, Mycol. Res. 95(1): 106 (1991). (Fig. 7a y 7b). 
Colonias dispersas, cafés pálidas. Conidióforos macronematosos, solitarios o en fascículos de 2-3, erectos, de rectos a ligeramente flexuosos, simples, pared lisa y gruesa, septados, cafés en la base y más pálidos hacia el ápice, hasta de $250 \mu \mathrm{m}$ de largo, de 6-8 $\mu \mathrm{m}$ de ancho, aproximadamente con $5 \mu \mathrm{m}$ de ancho en el ápice. Células conidiógenas polifialídicas, integradas, terminales, hasta con 4 proliferaciones sucesivas, con collarines en forma de embudo, de 3-5 $\mu \mathrm{m}$ de ancho y 3-5 $\mu \mathrm{m}$ de profundidad. Conidios agregados en masas mucilaginosas, acropleurógenos, elipsoidales, papilados, gutulados, pared lisa, sin septos, hialinos, de 13-15 × 7-8 $\mu \mathrm{m}$, con una sétula en cada extremo de $5 \mu \mathrm{m}$ de largo.

Material estudiado: sobre hojas muertas, col. G. Heredia, 10/06/95, CB850, CB850-1 y CB850-2. Rancho Guadalupe, mpio. Xalapa.

Distribución y sustratos: este hongo solamente se había hallado con anterioridad sobre ramas muertas de una angiosperma no identificada en Malasia (Kuthubutheen y Nawawi, 1991).

Dictyosporium digitatum J.L. Chen, C.H. Hwang \& Tzean, Mycol. Res. 95(9): 1145 (1991). (Fig. 8).

Colonias formadas por esporodoquios. Esporodoquios agrupados, superficiales o inmersos, globosos a subglobosos, elipsoidales, fusiformes o de forma irregular. Conidióforos micronematosos, erectos, rectos, simples o ramificados, pared lisa, septos constreñidos, de hialinos a cafés. Células conidiógenas monoblásticas, integradas, terminales, determinadas, subglobosas, doliiformes, cuneiformes, pared lisa y delgada, de hialinas a cafés, de 5-9 $\mu \mathrm{m}$ de largo y alrededor de $3 \mu \mathrm{m}$ de ancho. Conidios solitarios, acrógenos, quiroides, pared lisa y delgada, anaranjado grisáceos, dorado rojizos o café rojizos, de 68-88 $\times$ 33-37 $\mu \mathrm{m}$, con 5-7 hileras paralelas aplanadas y 57-88 células conidiales, cada hilera con 7-13 septos constreñidos, de 3-8.8 $\mu \mathrm{m}$ de ancho, células terminales en forma de dedos, rectas o flexuosas, recurvadas.

Material estudiado: hojas de Macadamia sp. col. G. Rosas, 13/06/95 CB851 y CB851-1. Rancho Guadalupe, mpio. Xalapa.

Distribución y sustratos: se ha encontrado creciendo sobre tallos caídos de restos herbáceos en Taiwán (Chen et al., 1991); sobre Machilus velutina, Pandanus furcatus, Phoenix hanceana, Pinus massoniana y en madera sumergida en Hong Kong y China (Lu et al., 2000; Zhuang, 2001, Tsui et al., 2001a, 2001b; Ho et al., 2002; Jiang et al., 2008) y en Licuala longicalycata en Tailandia (Pinruan et al., 2007). 
Endophragmiella collapsa (B. Sutton) S. Hughes, Fungi Canadenses, Ottawa 126 (1978). (Fig. 9).

$\equiv$ Endophragmia collapsa B. Sutton, Mycol. Pap. 132: 54 (1973).

Colonias dispersas, velutinas, cafés pálidas. Conidióforos macronematosos, gregarios, solitarios o en grupos, erectos, rectos o flexuosos, simples, subcilíndricos, pared lisa y delgada, septados, de color café dorado, más pálido hacia el ápice, hasta de $107 \mu \mathrm{m}$ de largo, de 3.5-5 $\mu \mathrm{m}$ de ancho en la base. Células conidiógenas monoblásticas, integradas, terminales, percurrentes. Conidios solitarios, acrógenos, ovoides a elipsoidales, pared gruesa y lisa, la mayoría con 2 septos ligeramente constreñidos, de color café pálido a café oscuro, célula basal más pálida, de 14.5-20 $\times 8-10 \mu \mathrm{m}$.

Material estudiado: hojas muertas de cafeto, col. J. Mena-Portales, 10/06/95, CB360. Rancho Guadalupe, mpio. Xalapa.

Distribución y sustratos: se ha colectado sobre troncos caídos y corteza de Acer sp., Alnus sp. y Salix sp. en Canadá (Sutton, 1973; Ellis, 1976 y Hughes, 1978a); y en troncos caídos de Salix sp. en la antigua Checoslovakia (Holubová-Jechová, 1986).

Endophragmiella valdiviana (Speg.) S. Hughes, New Zealand J. Bot. 17(2): 157 (1979). (Fig. 10).

$\equiv$ Helminthosporium valdivianum Speg., Rev. Fac. Agron. Univ. Nac. La Plata 6(1): 192 (1910).

इSporidesmium valdivianum (Speg.) M.B. Ellis, Mycol. Pap. 87: 29 (1963).

Colonias dispersas, velutinas, negras. Conidióforos macronematosos, solitarios, mayormente en fascículos, erectos, flexuosos, simples, septados, café rojizos oscuros, más pálidos hacia el ápice, hasta de $441 \mu \mathrm{m}$ de largo, de 18-23 $\mu \mathrm{m}$ de ancho. Células conidiógenas monoblásticas, integradas, terminales, percurrentes. Conidios solitarios, acrógenos, rectos o curvos, subfusiformes, truncados en la base, pared lisa, con 5-8(7) septos transversales y en ocasiones 1 septo longitudinal, cafés oscuros, células de los extremos subhialinas, de 34-63 × 11-18 $\mu \mathrm{m}$.

Material estudiado: sobre hojas de Macadamia sp., col. G. Heredia, 13/06/95, CB852, CB852-1 y CB852-2. Rancho Guadalupe, mpio. Xalapa.

Distribución y sustratos: se ha encontrado en ramas de Sophora microphylla en Chile (Ellis, 1963); sobre ramas de Macropiper excelsum en Nueva Zelanda y en Ulex europaeus en Inglaterra (Hughes, 1979) también ha sido colectada en madera podrida y hojas de un árbol no identificado en Japón (Matsushima, 1983). 
Epicoccum nigrum Link, Magazin Ges. Naturf. Freunde, Berlin 7: 32 (1815). (Fig. 11).

=Epicoccum purpurascens Ehrenb., Flora Berol., 2 (Crypt.): 136 (1818).

=Epicoccum vulgare Corda, Icon. fung. (Abellini) 1: 5 (1837).

=Phoma epicoccina Punith., M.C. Tulloch \& C.M. Leach, Trans. Br. Mycol. Soc. 59(2): 341 (1972).

=Toruloidea tobaica Svilv., Arch. Hydrobiol. 14, Suppl. 3: 519 (1936).

Colonias formadas por esporodoquios. Esporodoquios pulvinados, negros, dispersos en el sustrato. Conidióforos macronematosos o semimacronematosos, erectos, rectos, simples o ramificados, pared lisa o verrugosa, subhialinos a cafés pálidos, de 5-15 $\mu \mathrm{m}$ de largo, de 3-6 $\mu \mathrm{m}$ de ancho. Células conidiógenas monoblásticas, integradas, terminales, determinadas, cilíndricas. Conidios solitarios, acrógenos, subesféricos o piriformes, muriformes, cafés oscuros dorados, con una célula basal pedicelada, de 24.5-30 $\mu \mathrm{m}$ de diámetro.

Material estudiado: sobre hojas de Senecio sp., col. J. Mena-Portales, 06/07/95, CB493-5; sobre tallo de gramínea, col. J. Mena-Portales, 24/07/95, CB481-1. Xicochimalco, mpio. Xicochimalco.

Distribución y sustratos: E. nigrum es una especie saprobia cosmopolita, habita tanto en ambientes naturales como transformados; se ha aislado de restos de plantas, aire, suelo, animales, frutos, textiles, etc. (Ellis, 1971 y Farr et al., 2008).

Fusariella intermedia Mouch. \& Nicot, Revue Mycol., Paris 37(3): 181 (1973). (Fig. 12a y 12b).

Colonias dispersas, pulverulentas, negras. Conidióforos semimacronematosos, solitarios, erectos, rectos, irregularmente ramificados, pared lisa, subhialinos a oliváceos, hasta de $70 \mu \mathrm{m}$ de largo. Células conidiógenas monofialídicas, discretas, determinadas, curvas, cilíndricas, pared lisa o con ligeras rugosidades, subhialinas a oliváceas. Conidios catenulados, acrógenos, curvos y truncados en la base, pared lisa, 3 septos, constreñidos en los septos, grisáceos, más oscuros hacia la base, de $14-22 \times 4.5-7 \mu \mathrm{m}$.

Material estudiado: hojas en descomposición, col. G. Heredia, 06/07/95, CB853. Emiliano Zapata, mpio. Xalapa.

Distribución y sustratos: Fusariella intermedia fue descrita de muestras de suelo colectadas al norte de África (Mouchacca y Nicot, 1972); desde su descripción original no había sido registrada. 
Helicoma palmigenum (Penz. \& Sacc.) Linder, Ann. Mo. Bot. Gard. 16: 306 (1929). (Fig. 13).

Colonias dispersas, café grisáceas. Conidióforos macronematosos, solitarios, erectos, rectos, simples, simpodiales hacia la parte superior, geniculados, septados, café grisáceos, hasta de $285 \mu \mathrm{m}$ de largo, de 7-13 $\mu \mathrm{m}$ de ancho. Células conidiógenas monoblásticas, integradas, terminales, cilíndricas, denticuladas; dientecillos cilíndricos. Conidios solitarios, acrógenos, helicoidales, 11-16 septos, cafés oscuros, de 32-37 $\mu \mathrm{m}$ de diámetro; filamento conidial $13 / 4-2$ vueltas, de $10-11 \mu \mathrm{m}$ de grosor, cicatriz basal oscura.

Material estudiado: hojas, col. G. Heredia, 10/06/95, CB855. Coatepec, mpio. Coatepec.

Distribución y sustratos: esta especie se ha colectado en diversos sustratos; en hojas de Arenga engleri y sobre Cocos nucifera en Australia, Papua-Nueva Guinea y Taiwán (Matsushima, 1971, 1980; Goos, 1986; Taylor y Hyde, 2003); creciendo sobre hojas de Cocos nucifera y hojas de palma e inflorescencia de Roystonea en Hawai (Goos, 1986); sobre rama muerta de Syzygium jambos y Talipariti elatum en Cuba (Delgado-Rodríguez et al., 2002; Delgado-Rodríguez y Mena-Portales, 2004) y sobre hojas de Satakentia liukivensis en Japón (Tsui et al., 2006 y Tsui y Berbee, 2006).

Helminthosporium solani Durieu \& Mont., Explor. Sci. Algérie 1: 356 (1849). (Fig. 14). $\equiv$ Brachysporium solani (Durieu \& Mont.) Sacc., Syll. Fung. (Abellini) 4: 428 (1886). =Dematium atrovirens Harz, Bull. Soc. Imp. Nat. Moscou 44: 129 (1871). $=$ Helminthosporium atrovirens (Harz) E.W. Mason \& S. Hughes, in Hughes, Can. J. Bot. 31(5): 631 (1953).

=Spondylocladium atrovirens (Harz) Harz ex Sacc., Syll. Fung. (Abellini) 4: 483 (1886).

Colonias velutinas, cafés oscuras a negras. Conidióforos macronematosos, solitarios, erectos, rectos o flexuosos, simples con pared lisa o a veces verrugosa, cafés oscuros, más pálidos hacia el ápice, hasta de $450 \mu \mathrm{m}$ de largo, de 10-22 $\mu \mathrm{m}$ de ancho cerca de la base y alrededor de $9 \mu \mathrm{m}$ en el ápice. Células conidiógenas politréticas, integradas, terminales o intercalares, determinadas, cilíndricas. Conidios solitarios, acropleurógenos, obclaviformes, cafés pálidos a cafés, 2-8 distoseptos, de 29-46.5 $\times$ 9-13 $\mu \mathrm{m}$ en la parte más ancha, de 2-5 $\mu \mathrm{m}$ de grosor en el ápice, lisos, con una cicatriz basal oscura. 

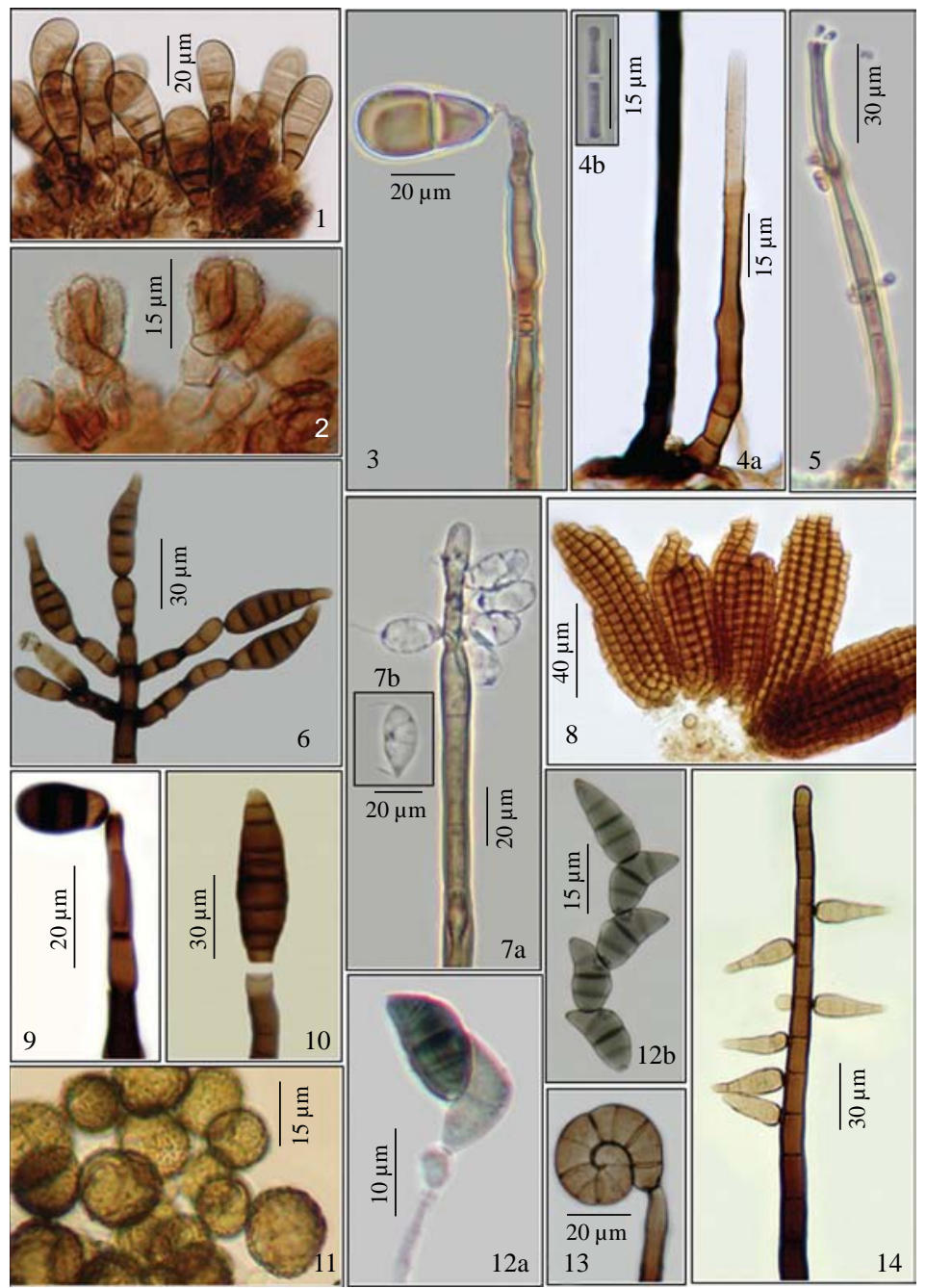

Figs. 1-14. 1. Bactrodesmium spilomeum: conidios claviformes. 2. Cheiromyceopsis echinulata: conidios equinulados. 3. Brachysporium obovatum: conidióforo con conidio. 4. Chalara aspera: a. seta y conidióforo, b. conidios piriformes 5. Chloridium virescens var. chlamydosporum: conidióforo. 6. Dendryphiopsis arbuscula: conidióforo ramificado con conidios fusiformes. 7. Dictyochaeta tumidospora: a. conidióforo y conidios, b. conidio maduro con sétulas. 8. Dictyosporium digitatum: conidios quiroides. 9. Endophragmiella collapsa: conidióforo con conidio. 10. Endophragmiella valdiviana: conidióforo simple y conidio subfusiforme. 11. Epicoccum nigrum: conidios muriformes. 12. Fusariella intermedia: a. conidióforo y conidios, b. conidios catenulados. 13. Helicoma palmigenum: conidio helicoidal adherido al conidióforo. 14. Helminthosporium solani: conidióforo con conidios obclaviformes. 
Material estudiado: sobre ramas muertas, col. G. Heredia, 10/06/95, CB356-2. Rancho Guadalupe, mpio. Xalapa.

Distribución y sustratos: esta especie tiene una amplia distribución y se ha registrado como causante de enfermedades en diversas plantas pero fundamentalmente en especies del género Solanum (Ellis, 1971; Matsushima, 1975; Dennis, 1986; Mercado-Sierra, 1984; Mendes et al., 1998; Delgado-Rodríguez et al., 2002; Cho y Shin, 2004 y Farr et al., 2008).

Hermatomyces tucumanensis Speg., Anal. Mus. Nac. Hist. Nat. Buenos Aires 13: 446 (1911). (Fig. 15).

Colonias dispersas, velutinas, cafés oscuras a café negruzcas. Conidióforos micronematosos, erectos, rectos o flexuosos, simples, lisos, sin septos, cafés pálidos, de $14.5 \mu \mathrm{m}$ de largo por 2.5-3 $\mu \mathrm{m}$ de ancho. Células conidiógenas monoblásticas, integradas, terminales, determinadas, cilíndricas. Conidios solitarios, acrógenos, lenticulares, elípticos o subesféricos en un plano, lisos, muriformes, células periféricas subhialinas, células centrales de cafés oscuras a negras, de 30-39.5 $\times 24-30 \mu \mathrm{m}$.

Material estudiado: sobre ramas muertas, col. G. Heredia, 10/06/95, CB359-1, CB362-1. Rancho Guadalupe, mpio Xalapa.

Distribución y sustratos: Hermatomyces tucumanensis se ha encontrado sobre ramas de Alchornea cordifolia, Averrhoa carambola, Coffea liberica y Elaeis en Argentina, Ghana y Sierra Leona (Ellis, 1971); creciendo sobre el pecíolo de la hoja muerta de Roystonea regia en Cuba (Mercado-Sierra, 1984); también se ha colectado en pecíolos de palma, ramas muertas y hojas de un árbol no identificado en Perú (Matsushima, 1993); sobre ramas muertas en Taiwán (Chang, 1995) y en Pandanus furcatus en Hong Kong (Lu et al., 2000; Zhuang, 2001).

Hyphodiscosia queenslandica Matsush., Matsushima. Mycol. Mem. 24 (1989). (Fig. 18a y 18b).

Colonias dispersas, grisáceas. Conidióforos macronematosos, solitarios, erectos, rectos, simples, septados, subhialinos con el ápice hialino, de 145-212 $\mu \mathrm{m}$ de largo, de 4-6 $\mu \mathrm{m}$ de ancho. Células conidiógenas integradas, terminales, poliblásticas, claviformes, denticuladas; dientecillos cilíndricos, truncados en el ápice, hialinos. Conidios solitarios, acrógenos, fusiformes o algunas veces elipsoidales, base cónico truncada, pared lisa, sin septos, hialinos, de 14.5-18 $\times$ 5-7 $\mu \mathrm{m}$; con una sétula filiforme en cada extremo, de 5-10 $\mu \mathrm{m}$ de largo. 
Material estudiado: sobre hojas caídas, col. G. Heredia, 10/06/09, CB858, CB858-1 y CB858-2. Xicochimalco, mpio. Xicochimalco.

Distribución y sustratos: este hongo sólo había sido registrado sobre hojas muertas de Cryptocarya mackinnoniana para Australia (Matsushima, 1989).

Kendrickiella phycomyces (Auersw.) K. Jacobs \& M.J. Wingf., in Jacobs, Wingfield, Jacobs \& Wingfield, Can. J. Bot. 79(1): 113 (2001). (Fig. 23).

$\equiv$ Graphium phycomyces (Auersw.) Sacc., Syll. Fung. (Abellini) 4: 614 (1886).

$\equiv$ Hantzschia phycomyces Auersw., Hedwigia 2 (1862).

ELeptographium phycomyces (Auersw.) Grosmann, Hedwigia 72: 193 (1932).

三Scopularia phycomyces (Auersw.) Goidànich, G., Boll. Staz. Patol. Veg. Roma 16:

1 (1935).

इPhialocephala phycomyces (Auersw.) W.B. Kendr., Can. J. Bot. 42: 1292 (1964).

Colonias dispersas, cafés pálidas. Conidióforos macronematosos, solitarios, erectos, rectos, ramificados, pared lisa, de color café oscuro a café negruzco, hasta de $150 \mu \mathrm{m}$ de largo. Células conidiógenas monofialídicas, discretas, terminales, determinadas, dispuestas en penicilos, subuladas, hialinas. Conidios endógenos, elipsoidales u obovoides, hialinos, lisos, de 4.4-5.5 × $1.5 \mu \mathrm{m}$.

Material estudiado: sobre hojarasca, col. J. Mena-Portales, 13/06/95, CB861 y CB861-1. Rancho Guadalupe, mpio. Xalapa.

Distribución y sustratos: existen registros sobre troncos muertos en Costa Rica (Morris, 1972); en barriles de roble y diferentes tipos de suelo en Congo y Jamaica (Ellis, 1976); también ha sido aislado de suelo forestal en Taiwán (Matsushima, 1980) y colectado sobre tallos muertos de Saccharum sp. en Cuba (Castañeda, 1986; Mercado-Sierra, et al., 1997).

Lauriomyces heliocephalus (V. Rao \& de Hoog) R.F. Castañeda \& W.B. Kendr., Univ. Waterloo Biol. Ser. 32: 26 (1990). (Fig. 16).

EHaplographium heliocephalum V. Rao \& de Hoog, Stud. Mycol. 28: 56 (1986).

Colonias dispersas, cafés oscuras. Conidióforos macronematosos, solitarios o en pequeños grupos, erectos, rectos o ligeramente curvos, simples, pared lisa y gruesa, cafés oscuros, de 60-87 $\mu \mathrm{m}$ de largo, aproximadamente de $6 \mu \mathrm{m}$ de ancho cerca de la base y $4 \mu \mathrm{m}$ cerca del ápice. Células conidiógenas poliblásticas, discretas, terminales, pared lisa, en ramas verticiladas, dispuestas en fascículos, hialinas. 
Conidios en cadenas acrópetas, acrógenos, rectos con un eje principal compuesto de 6-9 conidios, cilíndricos, en ocasiones redondeados en ambos extremos, pared delgada, hialinos, de 4-6 × 1.2-1.5 $\mu \mathrm{m}$.

Material estudiado: hojas muertas, col. G. Heredia, 28/06/95, CB859, CB859-1 y CB859-2. Rancho Guadalupe, mpio. Xalapa.

Distribución y sustratos: además del material tipo, colectado sobre hojas muertas de Alchornea latifolia en Cuba (Castañeda y Kendrick, 1990), sólo se había encontrado con anterioridad sobre hojas de Alchornea triplinervia en Brasil (Grandi y Atilli, 1996).

Melanocephala australiensis (G.W. Beaton \& M.B. Ellis) S. Hughes, New Zealand J. Bot. 17(2): 169 (1979). (Fig. 17).

$\equiv$ Endophragmia australiensis G.W. Beaton \& M.B. Ellis, in Ellis, Mycol. Pap. 106: 54 (1966).

Colonias dispersas, cafés pálidas. Conidióforos macronematosos, micronematosos, solitarios, dispersos u ocasionalmente en grupos, erectos, rectos, simples, de color café pálido, hasta de $150 \mu \mathrm{m}$ de largo y alrededor de $5 \mu \mathrm{m}$ de ancho, base bulbosa. Células conidiógenas monoblásticas, integradas, terminales, percurrentes. Conidios solitarios, acrógenos, obovoides, elipsoidales, con la base truncada, pared lisa, 2-3 septos, de color café muy oscuro casi negro y opacos, de 26.5-34.5 × 20-23.5.

Material estudiado: tronco muerto, col. J. Mena-Portales, 10/06/95, CB373-1; fruto muerto de Quercus col. J. Mena-Portales, 13/06/95, CB413-2; rama muerta, col. G. Rosas, 13/06/95, CB414-1; hojarasca de Quercus, col. G. Rosas, 13/06/95, CB419-6. Rancho Guadalupe, mpio. Xalapa.

Distribución y sustratos: se ha encontrado en una gran variedad de sustratos para Nueva Zelanda (Hughes, 1979), además sobre ramas muertas de un árbol no identificado en Cuba (Holubová-Jechová y Mercado-Sierra, 1984); en Podocarpus totara en Inglaterra (Kirk, 1986) y sobre troncos sumergidos en Australia (Hyde y Goh, 1998).

Menisporopsis novae-zelandiae S. Hughes \& W.B. Kendr., New Zealand J. Bot. 6: 369 (1968). (Fig. 22a y 22b).

Colonias anfígenas, irregulares, café oliváceas. Sinemas de 85.5-122 $\mu \mathrm{m}$ de largo, con una seta. Setas erectas, simples, subuladas, septadas, cafés oscuras, de 
250-300 $\mu \mathrm{m}$ de largo. Conidióforos macronematosos, rectos, septados, cafés pálidos, hasta de $122 \mu \mathrm{m}$ de largo. Células conidiógenas polifialídicas con varios collarines, collarines pequeños, integradas, determinadas, hialinas a cafés pálidas. Conidios agregados en una masa mucilaginosa, acrógenos, alantoides, pared lisa, 1 septo medio, hialinos, de 14.5-18 × 3-3.5 $\mu \mathrm{m}$; con una sétula en cada extremo.

Material estudiado: hojarasca de Quercus xalapensis y Q. germana, col. G. Rosas, 13/06/95, CB411-8; frutos muertos, col. J. Mena-Portales, 13/06/95, CB418-2; hojarasca de Quercus, col. G. Rosas, 13/06/95, CB420-3. Rancho Guadalupe, mpio. Xalapa.

Distribución y sustratos: existen colectas sobre hojas muertas de Cinnamomum spp., Cryptocarya mackinnoniana, Litsea japonica y Pasania spp. en Australia, Japón, Taiwán (Matsushima, 1975, 1980, 1989, 1993); en hojas de Beilschmiedia tarairi, Knightia excelsa y Rhopalostylis sp. en Nueva Zelanda (Hughes y Kendrick, 1968; McKenzie et al., 1992, 2004) y sobre hojas de Nectandra sp. y Ocotea spp. en Cuba (Castañeda et al., 2001; Delgado-Rodríguez et al., 2002 y Delgado-Rodríguez y Mena-Portales, 2004).

Metulocladosporiella musae (E.W. Mason) Crous, Schroers, J.Z. Groenew., U. Braun \& K. Schub., Mycol. Res. 110(3): 264-275 (2006). (Fig. 21a y 21b).

三Cladosporium musae E.W. Mason, in Martyn, Mycol. Pap. 13: 2 (1945).

EPericoniella sapientumicola Siboe, Afr. J. Mycol. Biotechnol. 2(1): 4 (1994).

Colonias dispersas o punctiformes, aterciopeladas, café grisáceas a café negruzcas. Conidióforos macronematosos o semimacronematosos, solitarios, erectos, rectos a ligeramente flexuosos, ramificados hacia el ápice, pared lisa, cafés a café negruzcos, pálidos hacia la parte media, hasta de $200 \mu \mathrm{m}$ de largo, de $3.5 \mu \mathrm{m}$ de ancho y alrededor de $12 \mu \mathrm{m}$ en la base, ramas hasta de $45 \mu \mathrm{m}$ de largo. Células conidiógenas poliblásticas, integradas, terminales e intercalares, simpodiales, con cicatrices prominentes. Conidios catenulados, acropleurógenos, cilíndricos, elipsoidales o fusiformes, pared lisa, 0-1 septo, subhialinos, de 7.5-11 $\times$ 2.5-3.5 $\mu \mathrm{m}$.

Material estudiado: hojas en descomposición, col. J. Mena-Portales, 13/06/95, CB849, CB849-1 y CB849-2. Rancho Guadalupe, mpio. Xalapa.

Distribución y sustratos: se ha encontrado ocasionando manchas foliares en hojas de Musa paradisiaca, M. paradisiaca var. sapientum, M. schweinfurthii y Musa spp. Existen registros de la presencia de esta especie en Ghana, Hong Kong, Islas Salomón, Jamaica, Nueva Guinea, Samoa, Sudan, Uganda, (Ellis, 1971); Hon- 


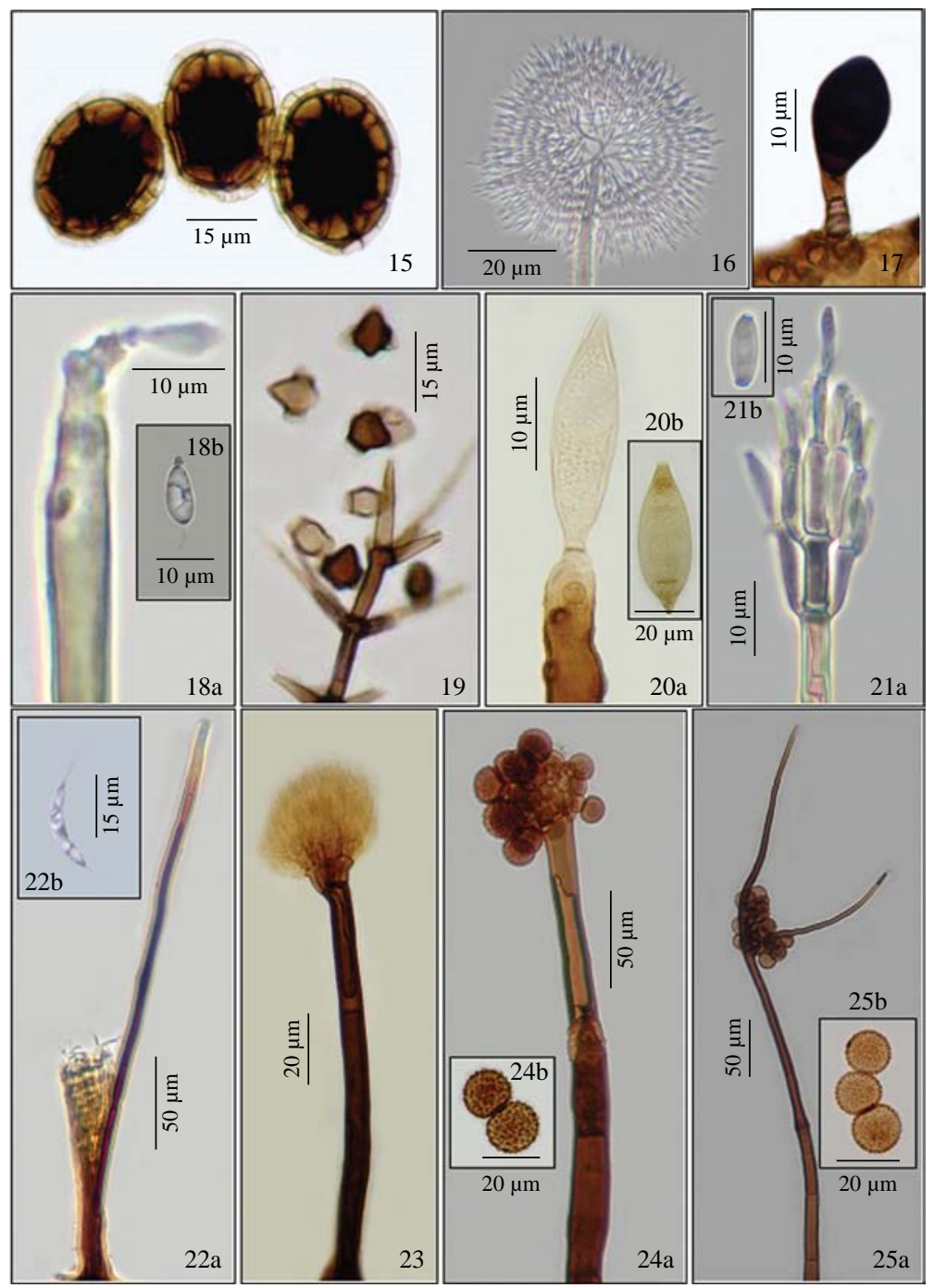

Figs. 15-25. 15. Hermatomyces tucumanensis: conidios con células periféricas. 16. Lauriomyces heliocephalus: conidióforo con conidios en cadenas. 17. Melanocephala australiensis: conidióforo con conidio obovoide. 18. Hyphodiscosia queenslandica: a. conidióforo con conidio inmaduro. b. conidio maduro con sétulas. 19. Physalidiella elegans: conidióforo y conidios con células laterales. 20. Pseudospiropes simplex: a. conidióforo con cicatrices, b. conidio navicular. 21. Metulocladosporiella musae: a. conidióforo con ramas, b. conidio. 22. Menisporopsis novae-zelandiae a. sinema y seta, b. conidio com setulas. 23. Kendrickiella phycomyces: conidióforo ramificado. 24. Periconia cookei: a. conidióforo, b. conidios verrugosos. 25. Periconia lateralis: a. conidióforo con una rama unilateral, b. conidios catenulados equinulados. 
duras (Ho et al., 1999); Sudáfrica (Siboe, 1994); Burundi, Camerún, Congo, Cuba, Ecuador, Egipto, Etiopía, Jamaica, Kenia y México (Crous et al., 2006).

Periconia cookei E.W. Mason \& M.B. Ellis, Mycol. Pap. 56: 72 (1953). (Fig. 24a y 24b).

Colonias dispersas, compactas, cafés pálidas. Conidióforos macronematosos, en grupos, erectos, rectos a ligeramente flexuosos, subulados, cafés pálidos, hasta de $610 \mu \mathrm{m}$ de largo, de 12-18 $\mu \mathrm{m}$ de ancho en la base, adelgazándose hacia la parte superior. Células conidiógenas monoblásticas, discretas, determinadas, terminales. Conidios catenulados, esféricos, verrugosos, cafés, de 15-18 $\mu \mathrm{m}$ de diámetro.

Material estudiado: sobre hojarasca, col. A. Mercado, 25/07/93. CB045. Rancho Guadalupe, mpio. Xalapa.

Distribución y sustratos: es común encontrar la en tallos de una gran variedad de plantas herbáceas; existen registros de la presencia de este hongo en Alemania (Mason y Ellis, 1953); Costa Rica, Belice (Morris, 1972, 1978); Estados Unidos de América (Huang y Hanlin, 1975; Morgan-Jones y Sinclair, 1978; Fell y Hunter, 1979); Hungría (Holuvobá-Jechová, 1979); Taiwán (Matsushima, 1980); India (Rao y Rao, 1964; Saikia y Sarbhoy, 1982; Grupta et al., 1983); Papua-Nueva Guinea (Shaw, 1984); Australia (Matsushima, 1989); Inglaterra (Taylor y Hyde, 2003); Cuba (Minter et al., 2001) y Rusia (Melnik y Popushoi, 1992 y Melnik et al., 2007).

Periconia lateralis Ellis \& Everh., J. Mycol. 2(9): 104 (1886). (Fig. 25a y 25b).

Colonias dispersas, compactas, café grisáceas. Conidióforos macronematosos, solitarios, erectos, rectos a ligeramente curvos, subulados, ápice setiforme estéril, cafés pálidos, hasta de $568 \mu \mathrm{m}$ de largo, de 14-23 $\mu \mathrm{m}$ de ancho en la base, adelgazándose hacia la parte superior. Células conidiógenas monoblásticas, discretas, determinadas, dispuestas en forma lateral, nacen directamente del estípite o de ramas unilaterales con ápices setiformes, esféricas o subesféricas. Conidios catenulados, esféricos, verrugosos a equinulados, cafés pálidos, de 13-15 $\mu \mathrm{m}$ de diámetro.

Material estudiado: sobre hojarasca, col. G. Heredia, 10/06/95. CB865. Rancho Guadalupe, mpio. Xalapa.

Distribución y sustratos: se ha aislado de suelo y restos vegetales, existen registros para Estados Unidos de América, Ghana, Hong Kong, India, Kenia, Mauritius, Nueva Guinea, Pakistán, Sierra Leona, Sudán, Uganda, Venezuela y Zambia (Ellis, 1971); Belice (Morris, 1978); Cuba (Mercado-Sierra, 1981, 1984); Malawi 
(Sutton, 1993); Argentina (Romero, 1998); Nigeria (Calduch et al., 2002) y Sudáfrica (Lee et al., 2004).

Physalidiella elegans (Mosca) Rulamort, Bull. Soc. Bot. Centre-Ouest, Nouv. sér. 21: 512 (1990). (Fig. 19).

EPhysalidium elegans Mosca, Allionia 11: 78 (1965).

Colonias efusas, delgadas, aracnoides o velutinas, gris oliváceas. Conidióforos macronematosos, erectos, rectos a flexuosos, ramificados, subulados, lisos, hasta de $300 \mu \mathrm{m}$ de largo, de 5-7 $\mu \mathrm{m}$ de ancho cerca de la base. Células conidiógenas monoblásticas, integradas, terminales, determinadas. Conidios solitarios, acrógenos, compuestos de una célula central obovoide o elipsoidal, café oscura, de 7-8.5 × 10 $\mu \mathrm{m}$ y dos células laterales subesféricas, hialinas o muy pálidas, de 2-4.5 × 4-6 $\mu \mathrm{m}$.

Material estudiado: semillas de encino, col. J. Mena-Portales, 13/06/95, CB413-1; hojas de Carpinus caroliniana, col. J. Mena-Portales, 13/06/95, CB860. Rancho Guadalupe, mpio. Xalapa.

Distribución y sustratos: a esta especie se le ha colectado sobre rastrojo de Triticum sp. en Italia (Ellis, 1971), en hojas de un árbol no identificado en Papua Nueva Guinea (Matsushima, 1971), sobre una palma en Tanzania (Pirozynski, 1972); creciendo en pecíolos de palma y frutos de Inga sp. en Perú (Matsushima, 1993) y en madera sumergida en Hong Kong (Zhuang, 2001).

Pseudospiropes simplex (Nees) M.B. Ellis, Dematiaceous Hyphomycetes (Kew): 260 (1971). (Fig. 20a y 20b).

$\equiv$ Helminthosporium simplex Kunze, in Nees \& Nees, Nova Acta Acad. Caes. Leop.Carol. Nat. Cur. Dresden 9: 241 (1818).

Teleomorfo: Strossmayeria bakeriana (Henn.) Iturr., Mycotaxon 36(2): 408 (1990).

Colonias dispersas, café oliváceas a café negruzcas. Conidióforos macronematosos, dispersos o agregados, erectos, rectos a flexuosos, simples, pared lisa, cafés oscuros, más pálidos en el ápice, hasta de $400 \mu \mathrm{m}$ de largo, de 4.5-6 $\mu \mathrm{m}$ de ancho, cicatrices oscuras, prominentes. Células conidiógenas poliblásticas, integradas, terminales, intercalares, simpodiales, cilíndricas a claviformes, cicatrizadas. Conidios solitarios, acropleurógenos, fusiformes o naviculares, oliváceos a café dorados pálidos, 6-11 pseudoseptos, de 26-44 × 9-13 $\mu \mathrm{m}$, de 2-3 $\mu \mathrm{m}$ de ancho en la cicatriz basal.

Material estudiado: tronco muerto, col. A. Mercado, 30/07/93, CB033; sobre rama muerta, col. A. Mercado, 20/05/94, CB035. Jardín Botánico Francisco Javier 
Clavijero, mpio. Xalapa; sobre gramínea, col. A. Mercado, 20/05/94, CB263-1.Volcán San Martín, mpio. San Andrés Tuxtla; sobre bejuco, col. G. Rosas, 16/06/95, CB473-2. Comapa, mpio. Comapa.

Distribución y sustratos: esta especie es común encontrarla sobre madera muerta y diversas plantas; existen registros de colectas en Canadá (Hughes y Cooke, 1979; Ginns, 1986; Sutton, 1973); Inglaterra (Dennis, 1986); Cuba (Mercado-Sierra y Mena-Portales, 1986; Holubová-Jechová y Mercado-Sierra, 1989); Perú, Sudáfrica y Taiwán (Matsushima, 1987, 1993, 1996; Lee et al., 2004); Hong Kong (Lu et al., 2000); Nueva Zelanda (Hughes, 1978b; McKenzie et al., 2000); Rusia (Melnik et al., 2007) y China (Zhuang, 2001; Shang y Zhang, 2007).

\section{AGRADECIMIENTOS}

Se agradece el apoyo de las siguientes instituciones: Comisión Nacional para el Conocimiento y uso de la Biodiversidad (proyectos P030 y B139), Instituto de Ecología A.C. y la red Iberoamericana sobre Diversidad, Ecología y Usos de los Hongos Microscópicos del Programa Iberoamericano de Ciencia y Tecnología para el Desarrollo (Programa CYTED).

\section{LITERATURA CITADA}

Calduch, M., J. Gené, J. Guarro, A. Mercado-Sierra y R. F. Castañeda. 2002. Hyphomycetes from Nigerian rain forest. Mycologia 94(1): 127-135.

Castañeda, R. F. 1986. Deuteromycotina de Cuba. Hyphomycetes IV. Instituto de Investigaciones Fundamentales en Agricultura Tropical "Alejandro Humboldt”. La Habana. 17 pp.

Castañeda, R. F. y B. Kendrick. 1990. Conidial fungi from Cuba: I. Univ. Waterloo Biol. Ser. 32: 1-53.

Castañeda, R. F., T. Iturriaga, M. Saikawa, J. Cano y J. Guarro. 2001. The genus Menisporopsis in Venezuela with the addition of $M$. anisospora anam. sp. nov. from a palm tree. Cryptog. Mycol. 22: 259-263.

Chang, H. S. 1995. Notes on Taiwan dematiaceous hyphomycetes, some species of the genera Exserticlava, Craspedodidymum and Hermatomyces Bot. Bull. Acad. Sin. 36: 243-246.

Chen, J. L., C. H. Hwang y S. S. Tzean. 1991. Dictyosporium digitatum, a new hyphomycete from Taiwan. Mycol. Res. 95: 1145-1149.

Cho, W. D. y H. D. Shin. 2004. List of plant diseases in Korea. 4th ed. Korean Society of Plant Pathology. Seoul, Korea. 779 pp. 
Chlebicki, A. y M. A. Chmiel. 2006. Microfungi of Carpinus betulus from Poland I. Annotated list of microfungi. Acta Mycol. 41(2): 253-278.

Crous, P. W., H. J. Schroers, J. Z. Groenewald, U. Braun y K. Schubert. 2006. Metulocladosporiella gen. nov. for the causal organism of Cladosporium speckle disease of banana. Mycol. Res. 110: 264-275.

Delgado-Rodríguez, G., J. Mena-Portales, M. Calduch y C. Decock. 2002. Hyphomycetes (hongos mitospóricos) del área protegida Mil Cumbres, Cuba Occidental. Cryptogam. Mycol. 23: 277-293.

Delgado-Rodríguez, G. y J. Mena-Portales. 2004. Hifomicetos (hongos anamórficos) de la Reserva Ecológica ”Alturas de Banao” (Cuba). Bol. Soc. Micol. Madrid 28: 115-124.

Delgado-Rodríguez, G., G. Heredia, R. M. Arias y J. Mena-Portales. 2006. Contribución al estudio de los hongos anamórficos de México. Nuevos registros para el estado de Veracruz. Bol. Soc. Micol. Madrid 30: 235-242.

Dennis, R. W. G. 1986. Fungi of the Hebrides. Royal Botanic Gardens. Kew 383 pp.

Ellis, M. B. 1958. Clasterosporium and some allied Dematiaceae Phragmosporae I. Mycol. Pap. 70: 90.

Ellis, M. B. 1959. Clasterosporium and some allied Dematiaceae Phragmosporae II. Mycol. Pap. 72(9): 76.

Ellis, M. B. 1963. Dematiaceous Hyphomycetes. Commonwealth Mycological Institute. Kew. 608 pp.

Ellis, M. B. 1971. Dematiaceous Hyphomycetes IV. Mycol. Pap. 87: 42

Ellis, M. B. 1976. More Dematiaceous Hyphomycetes. Commonwealth Mycological Institute. Kew. 507 pp.

Farr, D. F., A. Y. Rossman, M. E. Palm y E. B. McCray. Fungal databases, Systematic Mycology and Microbiology Laboratory, ARS, USDA. Retrieved May 1, 2008 http:// nt.ars-grin.gov/fungaldatabases/

Fell, J. W. y I. L. Hunter. 1979. Fungi associated with the decomposition of the black rush, Juncus roemerianus, in south Florida. Mycologia 71: 322-342.

Gams, W. y V. Holobová-Jechová. 1976. Chloridium and some other dematiaceous hyphomycetes growing on decaying wood. Stud. Mycol. 13: 21-24.

Ginns, J. H. 1986. Compendium of plant disease and decay fungi in Canada 1960-1980. Res. Br. Can. Agric. Publ. 1813: 416.

Goos, R. D. 1986. A review of the anamorph genus Helicoma. Mycologia 78: 744-761.

Grandi, R. A. P. 1991. Hyphomycetes descompositores 2. Táxons associados as raízes de Maranta bicolor Ker. Rev. Bras. Biol. 51(1): 133-141.

Grandi, R. A. P. 1999. Hyphomycetes descompositores do folhedo de Euterpe edulis Mart. Hoehnea 26(1): 87-101.

Grandi, R. A. P. y D. S. Attili. 1996. Hyphomycetes on Alchornea triplinervia (Spreng.) Muell. Arg. leaf litter from the Ecological Reserve Juréia-Itatins, State of Sao Paulo, Brazil. Mycotaxon 60: 373-386.

Grandi, R. A. P. y L. F. P. Gusmao. 1996. Hyphomycetes descompositores de raízes de Calathea zebrina (Sims) Lindl. (Marantaceae), provenientes da Reserva Biológica do Alto da Sierra de Paranapiacaba, Santo André, S. P., Brasil. Rev. Bras. Bot. 19(2): 165-172. 
Gupta, D., P. N. Chowdhry y B. Padhi. 1983. Some phytopathogenic fungi of ornamental plants from India. Indian Phytopathol. 36: 244-246.

Heredia, G. y M. Reyes. 1999. Hongos conidiales de bosque mesófilo: algunas especies foliícolas y de la hojarasca desconocidas para México. Rev. Mex. Micol. 15: 79-88.

Heredia, G., M. Reyes, R. M. Arias, J. Mena-Portales y A. Mercado-Sierra. 2004. Adiciones al conocimiento de la diversidad de los hongos conidiales del bosque mesófilo de montaña del estado de Veracruz. Acta Bot. Mex. 66: 1-22.

Heredia, G., R. M. Arias y J. Mena-Portales. 2006. Adiciones al conocimiento de la diversidad de los hongos conidiales del bosque mesófilo de montaña del estado de Veracruz II. Acta Bot. Mex. 77: 15-30.

Ho, W. H., R. F. Castañeda, F. M. Dugan y S. C Jong. 1999. Cladosporium and Cladophialophora in culture: descriptions and an expanded key. Mycotaxon 72: 115157.

Ho, W. H., Yanna, K. D. Hyde e I. J. Hodgkiss. 2002. Seasonality and sequential occurrence of fungi on wood submerged in Tai Po Kau Forest Stream, Hong Kong. Fungal Divers. 10: 21-43.

Holubová-Jechová, V. 1972. Lignicolous Hyphomycetes from Czechoslovakia 2. Bactrodesmium. Folia Geobot. 7: 407-418

Holubová-Jechová, V. 1979. Lignicolous and some other saprophytic Hyphomycetes from Hungary. Česká Mykol. 33(3): 139-149.

Holubová-Jechová, V. 1986. Lignicolous Hyphomycetes from Czechoslovakia. 8. Endophragmiella and Phragmocephala. Folia Geobot. 21: 173-197.

Holubová-Jechová, V. y A. Mercado-Sierra. 1984. Studies on Hyphomycetes from Cuba II. Hyphomycetes from the Isla La Juventud. Česká . Mykol. 38(2): 96-120.

Holubová-Jechová, V. y A. Mercado-Sierra. 1989. Hyphomycetes from Loma de la Coca and some localities of La Habana and Matanzas provinces, Cuba. Acta Bot. Cubana 76: 1-15.

Huang, L. H. y R. T. Hanlin. 1975. Fungi occurring in freshly harvested and in-market pecans. Mycologia 67: 689-700.

Hughes, S. J. 1978a. Endophragmiella collapsa. Fungi Canadenses 126: 1-2.

Hughes, S. J. 1978b. New Zealand fungi 25. Miscellaneous species. New Zealand J. Bot. 16: 311-370.

Hughes, S. J. 1979. Relocation of species of Endophragmia auct. with notes on relevant generic names. New Zealand J. Bot. 17: 139-188.

Hughes, S. J. y J. C. Cooke. 1979. Pseudospiropes simplex. Fungi Canadenses 145: 1-2.

Hughes, S. J. y W. B. Kendrick. 1968. Menispora, Codinaea, Menisporopsis. New Zealand J. Bot. 6: 323-375.

Hyde, K. D. y T. K. Goh. 1998. Fungi on submerged wood in Lake Barrine, north Queensland, Australia. Mycol. Res. 102: 739-749.

Jiang, M., M. Wongsawas, H. K. Wang, F. C. Lin y Y. C. Liang. 2008. Three new records of lignicolous freshwater Hyphomycetes from mainland, China. J. Agr. Technol. 4(1): 101-108.

Kirk, P. M. 1986. New or interesting microfungi XV. Miscellaneous Hyphomycetes from the British Isles. Trans. Brit. Mycol. Soc. 86: 409-428. 
Kuthubutheen, A. J. y A. Nawawi. 1991. Three new species of Dictyochaeta with non setose conidiophores and non septate setulate conidia from Malaysia. Mycol. Res. 95(1): 104-107.

Lee, S., V. Melnik, J. E. Taylor y P. W. Crous. 2004. Diversity of saprobic Hyphomycetes on Proteaceae and Restionaceae from South Africa. Fungal Divers. 17: 91-114.

Lu, B., K. D. Hyde, W. H. Ho, K. M. Tsui, J. E. Taylor, K. M. Wong, K. D. Yanna y D. Zhou. 2000. Checklist of Hong Kong fungi. Fungal Diversity Press. Hong Kong. 207 pp.

Mason, E. W. y M. B. Ellis. 1953. British species of Periconia. Mycol. Pap. 56. 127 pp.

Matsushima, T. 1971. Microfungi of the Solomon Islands and Papua-New Guinea. Published by the author. Kobe. 78 pp.

Matsushima, T. 1975. Icones microfungorum a Matsushima lectorum. Nippon Printing Publishing Co. Osaka. 209 pp.

Matsushima, T. 1980. Matsushima Mycological Memoirs No. 1. Matsushima Fungus Collect. Kobe. 82 pp.

Matsushima, T. 1983. Matsushima Mycological Memoirs No. 3. Matsushima Fungus Collect. Kobe. 90 pp.

Matsushima, T. 1987. Matsushima Mycological Memoirs No. 5. Matsushima Fungus Collect. Kobe. 100 pp.

Matsushima, T. 1989. Matsushima Mycological Memoirs No. 6. Matsushima Fungus Collect. Kobe. 100 pp.

Matsushima, T. 1993. Matsushima Mycological Memoirs No. 7. Matsushima Fungus Collect. Kobe. 75 pp.

Matsushima, T. 1996. Matsushima Mycological Memoirs No. 9. Matsushima Fungus Collect. Kobe. 40 pp.

McKenzie, E. H. C., P. J. O’Sullivan y J. P. Wilkie. 1992. A list of type specimens of New Zealand fungi held in DSIR Plant Protection Herbarium (PDD). Mycotaxon 43: 77156.

McKenzie, E. H. C., P. K. Buchanan y P. R. Johnston. 2004. Checklist of fungi on nikau palm (Rhopalostylis sapida and $R$. baueri var. chessemanii) in New Zealand. New Zealand J. Bot. 42: 335-355.

Melnik, V. A. y I. S. Popushoi. 1992. Imperfect fungi on species on trees and shrubs: An illustrated handbook. Akademiia Nauk Respubliki Moldova. Kishinev. 361 pp.

Melnik, V. A., E. S. Popov y D. A. Shabunin. 2007. Contributions to the studies of mycobiota in Novgorod and Pskov regions. I. Hyphomycetes. Mikol. Fitopatol. 41: 515-525.

Mena-Portales, J., J. Gené y J. Guarro. 2000. Contribución al estudio de los hifomicetos en España XV. Bol. Soc. Mic. Madrid 25: 73-82.

Mendes, M. A. S., V. L. da Silva y J. C. Dianese. 1998. Fungos em plants no Brasil. EmbrapaSPI/Embrapa-Cenargen. Brasilia. 555 pp.

Mercado-Sierra, A. 1981. Lista preliminar de hifomicetes dematiáceos de la Estación Ecológica de Sierra de Rosario y zonas adyacentes. Acta Bot. Cubana 6: 1-6.

Mercado-Sierra, A. 1984. Hifomicetes dematiáceos de Sierra del Rosario, Cuba. Academia de Ciencias de Cuba, Instituto de Botánica. La Habana. 181 pp.

Mercado-Sierra, A. y J. Mena-Portales. 1986. Hifomicetos de Topes de Collantes, Cuba I. Especies holoblásticas. Acta Bot. Hung. 32(1-4): 189-205. 
Mercado-Sierra, A., Holubová-Jechová y J. Mena-Portales. 1997. Hifomicetos dematiáceos de Cuba, Enteroblásticos. Monografie XXIII. Museo Regionale di Scienze Naturali. Torino. 388 pp.

Minter, D. W., M. Rodriguez y J. Mena-Portales. 2001. Fungi of the Caribbean: an annotated checklist. PDMS Publishing. Londres. 946 pp.

Morgan-Jones, G., y R. C. Sinclair. 1978. Fungi of Alabama. VII. Dematiaceous Hyphomycetes. J. Alabama Acad. Sci. 49: 1-15.

Morris, F. E. 1972. Costa Rican Hyphomycetes. Mycologia 64: 887-896.

Morris, F. E. 1978. Belizean Hyphomycetes. Mycotaxon 7: 265-274.

Mouchacca, J. y J. Nicot. 1972. Les Fusariella des sols arides. Rev. Mycol. 37(3): 168182.

Nag Raj, T. R. y W. B. Kendrick. 1975. A monograph of Chalara and allied genera. Wilfrid Laurier University Press. Ontario. 200 pp.

Pinruan, U., K. D. Hyde, S. Lumyong, E. H. C. McKenzie y E. B. G. Jones. 2007. Occurrence of fungi on tissues of the peat swamp palm Licuala longicalycata. Fungal Divers. 25: 157-173.

Pirozynski, K. A. 1972. Microfungi of Tanzania I. Miscellaneous fungi on oil palm II. New Hyphomycetes. Mycol. Pap. 129: 1-65.

Pirozynski, K. A. y C. S. Hodges. 1973. New Hyphomycetes from South Carolina. Can. J. Bot. 51: 157-173.

Rao, P. R. y D. Rao. 1964. The genus Periconia from India. Mycopathologia 22: 285-310.

Revay, A. 1986. Dematiaceous Hyphomycetes inhabiting forest debris in Hungary II. Studia Bot. Hung. 19: 73-78.

Romero, A. I. 1998. Clave de las especies de micromicetos xilófilos registrados sobre Eucalyptus viminalis Labill. en el noreste de la Provincia de Buenos Aires, Argentina. Bol. Soc. Micol. Madrid 23: 47-48.

Saikia, U. N. y A. K. Sarbhoy. 1982. Hyphomycetes of India V. The genus Periconia. Indian Phytopathol. 35: 277-281.

Shang, Z. Q. y X. G. Zhang. 2007. Taxonomic studies of Pseudospiropes from Yunnan, China. Mycotaxon 100: 149-153.

Shaw, D. E. 1984. Microorganisms in Papua New Guinea. Dept. Primary Ind. Res. Bull. 33: $1-344$.

Siboe, G. M. 1994. Taxonomy of the fungus causing speckling disease of bananas (Musa spp.) in Kenya. Afr. J. Mycol. Biotechnol. 2: 1-6.

Sutton, B. C. 1973. Hyphomycetes from Manitoba and Saskatchewan, Canada. Mycol. Pap. 132: 1-143.

Sutton, B. C. 1993. Mitosporic fungi from Malawi. Mycol. Pap. 167: 1-93.

Taylor, J. E. y K. D. Hyde. 2003. Microfungi of tropical and temperate palms. Fungal Diversity Press. Hong Kong. 459 pp.

Tidwell, T. E. 1990. Index of diseases and microorganisms associated with eucalyptus in California. State of California Department of Food and Agriculture. Sacramento. 55 pp.

Tokumasu, S., K. Tubaki y L. Manoch. 1990. A preliminary list of hyphomycetes isolated from pine leaf litter of Thailand. Rep. Tottori Mycol. Inst. 28: 185-190. 
Tsui, C. K. M. y M. L. Berbee. 2006. Phylogenetic relationships and convergence of helicosporous fungi inferred from ribosomal DNA sequences. Mol. Phylogenet. Evol. 39: 587-597

Tsui, C. K. M., K. D. Hyde y I. J. Hodgkiss. 2001a. Longitudinal and temporal distribution of freshwater ascomycetes and dematiaceous hyphomycetes on submerged wood in the Lam Tsuen River, Hong Kong. J. North Am. Benthol. Soc. 20(4): 533-549.

Tsui, C. K. M., K. D. Hyde y I. J. Hodgkiss. 2001b. Colonization patterns of wood-inhabiting fungi on baits in Hong Kong rivers with reference to the effects of organic pollution. Antoine van Leeuwenhoek 79: 33-38.

Tsui, C. K. M., S. Sivichai y M. L. Berbee. 2006. Molecular systematics of Helicoma, Helicomyces and Helicosporium and their teleomorphs inferred from rDNA sequences. Mycologia 98: 94-104.

Williams-Linera, G. 2007. El bosque de niebla del centro de Veracruz: Ecología, historia y destino en tiempos de fragmentación y cambio climático. Comisión Nacionaciol para el Conocimiento y Uso de la Biodiversidad - Instituto de Ecología, A. C. Xalapa, Veracruz. 204 pp.

Zhuang, W. Y. 2001. Higher fungi of tropical China. Mycotaxon. Ithaca. 485 pp. 